\title{
Paradiplomacia y relaciones internacionales: de la práctica hacia su curricularización en Argentina
}

\author{
Nahuel Oddone* \\ Florencia Rubiolo** \\ Mariana Calvento***
}

\section{RESUMEN}

La paradiplomacia, como práctica de gobiernos locales y regionales, es objeto de una multiplicidad de estudios en diferentes latitudes. Estos han contribuido a relevar y visibilizar el comportamiento de estos actores, en especial, su aporte a las relaciones internacionales y a la política exterior. Durante este proceso, se fue construyendo también una mayor conceptualización del término, que permite establecer diá- logos que comienzan a superar los estudios de caso y dan forma a un emergente campo formal de investigación. Estos avances consistieron en la inclusión formal de la temática en planes de estudios vinculados a las relaciones internacionales a través de un proceso heterogéneo. En este artículo se busca generar una contribución al estudio de la paradiplomacia en Argentina, desde una perspectiva regional, para potenciar los estudios académicos en este emergente campo de las relaciones internacionales.

Las opiniones aquí expresadas son de estricto carácter personal y pueden no representar a su institución de pertenencia.

Doctor en estudios internacionales por la Universidad del País Vasco/Euskal Herriko Unibertsitatea (UPv/EHu). Jefe de Promoción e Intercambio de Políticas Sociales Regiones del Instituto Social del Mercosur (ISM), Asunción, (Paraguay). [oddone.nahuel@gmail.com]; [https://orcid.org/0000-0002-3120-3914].

* Doctora en relaciones internacionales. Investigadora adjunta Conicet (CIECS). Directora del Doctorado en Relaciones Internacionales, Universidad Católica de Córdoba, Córdoba, (Argentina); [frubiolo@gmail.com]; [https:// orcid.org/0000-0002-5669-7332]

*** Doctora en ciencias políticas. Profesora adjunta (Unicen) e investigadora adjunta (Conicet). Ceipil-Unicen-Cic/ Conicet, Buenos Aires, (Argentina); [marianacalvento@yahoo.com.ar]; [https://orcid.org/0000-0002-0257-3270] Recibido: 24 de enero de 2020 / Modificado: 18 de febrero de 2020 / Aceptado: 28 de febrero de 2020

Para citar este artículo:

Oddone, N.; Rubiolo, F. y Calvento, M. (2020). Paradiplomacia y relaciones internacionales: de la práctica hacia su curricularización en Argentina. OASIS, 32, pp. 63-84.

Dor: https://doi.org/10.18601/16577558.n32.06 
Palabras clave: paradiplomacia, actores subestatales, desarrollo, enseñanza

\section{Paradiplomacy and international relations: From practice to its curricularization in argentina}

\section{ABSTRACT}

Paradiplomacy, as a practice of local and regional governments, is the subject of multiple studies in different latitudes. These have helped to systematize and to make visible the behavior of these actors, especially their contribution to international relations and foreign policy. During this process, a greater conceptualization of the term has also been constructed, allowing dialogues to begin to overcome case studies and shape an emerging formal field of research. These advances consisted on the formal inclusion of the subject in curricula linked to international relations through a heterogeneous process. This article seeks to generate a contribution to the study of paradiplomacy in Argentina, from a regional perspective, to enhance academic studies in this emerging field of International Relations.

Key words: paradiplomacy, substate actors, development, teaching

\section{INTRODUCCIÓN}

La paradiplomacia como práctica de las unidades subnacionales es crecientemente objeto de multiplicidad de estudios en diferentes latitudes. Estos análisis han contribuido a relevar y visibilizar el comportamiento de estos actores internacionales y su aporte a las relaciones internacionales y la política exterior. Durante este proceso de investigación colectivo transnacional, se fue construyendo una conceptualización del término a partir de diferentes experiencias empíricas que permiten establecer diálogos que superan los estudios de caso y dan forma a un emergente campo formal de investigación. La paradiplomacia, al igual que las relaciones internacionales, es abordada desde diferentes matrices disciplinarias.

Más allá de los análisis de caso materializados en diferentes investigaciones, estos avances consintieron la inclusión formal de la temática de la paradiplomacia en distintos planes de estudios vinculados a las relaciones internacionales; se trata de un proceso heterogéneo que ha adquirido características diferenciales a lo largo de América Latina, en general, y de Argentina, en particular. En este trabajo exploratorio se persigue comprender cuál ha sido la situación en Argentina y responder algunas preguntas que surgen de los debates académicos sobre la paradiplomacia como parte de la disciplina de las relaciones internacionales. En particular se buscará responder:

- ¿Por qué es relevante la inclusión de la paradiplomacia dentro del campo de estudio de las relaciones internacionales?

- ¿Dónde ha sido estudiada la paradiplomacia en Argentina? ¿En qué universidades nacionales y centros de investigación principalmente?

- ¿Cómo se ha visto reflejada la paradiplomacia en los planes de estudios de las carreras de relaciones internacionales de grado y posgrado en la Argentina? 
En dónde ha sido estudiada la paradiplomacia y el cómo ha sido estudiada en Argentina contribuyen a la revisión teórico-práctica del concepto. Este artículo de carácter exploratorio está organizado de la siguiente forma. Tras la introducción, en una primera parte se analiza brevemente el proceso de conceptualización del término de paradiplomacia en el marco de las relaciones internacionales; en una segunda parte, se relevan las universidades nacionales y centros de investigación en donde ha sido incluida y dictada, analizando cómo ha sido estudiada en Argentina a partir de su inclusión en los planes de estudio. Por último, se proponen algunas reflexiones.

\section{PARADIPLOMACIA: DE LA PRÁCTICA A LA CONCEPTUALIZACIÓN}

La conmemoración del centenario del nacimiento de la disciplina de las relaciones internacionales -aniversario ligado al fin de la primera guerra mundial, al inicio de las cátedras que estudiaban política internacional y al surgimiento de centros de investigación en los países más desarrollados-, la encuentra en un proceso de revisión y reinterpretación de sus propias imágenes fundacionales. Muchos de los mitos fundacionales permanecieron relativamente incuestionados hasta la introducción -reciente- de perspectivas más críticas y plurales vinculadas a los actores, los contextos, los temas y sus referentes, que han contribuido a la conformación del campo disciplinar.

La paradiplomacia, como acción internacional de los gobiernos locales y regionales, producto de un mandato electoral, se ha correspondido con los nuevos enfoques revisionistas que buscan comprender mejor los actores internacionales, como fuerzas materiales que coadyuvan en la construcción y el cambio del sistema internacional. Estos enfoques han ubicado la agencia del sujeto de investigación (actor internacional subnacional) comprendiendo su papel en la construcción de la estructura del sistema internacional dentro de la dualidad agencia-estructura. La paradiplomacia ha colaborado en pensar las estrategias de internacionalización de actores locales y regionales como política pública transversal y, en este proceso, fortalecido los poderes de agencia de las unidades subestatales del sistema internacional. Como sostienen Liu y Song (2020), el análisis del actorness con "el objetivo de comprender quién es un jugador internacional es una de los marcos téoricos más desarrollados para estudiar la paradiplomacia” (Liu y Song, 2020, p. 4).

Además, vale la pena mencionar que parte de los referentes que se han dedicado al estudio de la paradiplomacia están siendo revisitados, pues a partir de enfoques históricos se ha rescatado una generación perdida de internacionalistas previa a la gran guerra (Knutsen, 2008), que incluyó en sus estudios los aportes de los gobiernos locales para el internacionalismo (Luna Pont, 2019; Oddone y Luna Pont, 2019). El internacionalismo era interpretado como un factor de construcción civilizatoria en donde la cuestión urbana realizaba su aporte a través del movimiento municipalista que se fue nucleando a partir del Primer Congreso Internacional y Exposición de Ciudades realizado en Ghent, Bélgica, en 1913. También por aquellos años surgieron algunas organizaciones de carácter internacional que nucleaban 
a las ciudades con el objetivo de fortalecer los estudios de la administración pública local, la planificación urbana y la cooperación intermunicipal. Estos ejercicios de revisión aportan a una reinterpretación del origen del campo disciplinar.

Estudios más recientes que reclamaron más pluralismo y diversidad teórica (Acharya y Buzan, 2010; 2019) han adquirido una mayor centralidad impactando con fuerza en la reinterpretación de las relaciones internacionales, tanto en la periferia como en el centro, e hicieron un llamado a indagar en los márgenes de la propia disciplina. La paradiplomacia se encuentra aún en los márgenes de la disciplina, pero como ha mencionado recientemente Noé Cornago (2019), en un proceso de creciente acercamiento hacia el mainstream. Sin lugar a duda, la práctica de la paradiplomacia comenzó antes que su conceptualización.

Desde un enfoque histórico, la acción internacional de los gobiernos locales comenzó -incluso- antes de la conformación de los Estados modernos, en donde las ciudades tenían un papel importante por constituir ciudadesEstado durante la antigua Grecia, los reinos de Taifas del Al Andalus que también eran un conjunto de ciudades-Estado; o las ligas de ciudades como Liga Hanseática o las Repubbliche Marinare durante el medioevo, entre otros ejemplos. Aun después de la conformación del primer orden económico mundial que permitió comprender una nueva dimensión global y del surgimiento de los Estados modernos con la Paz de Westfalia que ponía fin a la guerra de los treinta años (1618-1648), persistieron expresiones históricas que demuestran el poder de lo local como sucede en los años previos a la unificación de la Repubblica Italiana (18611870) o de la Bundesrepublik Deutschland (1871) en donde se asistía a una fuerte fragmentación del poder de base territorial. Sin embargo, el desempeño internacional de actores subnacionales desde la etapa fundacional de la disciplina ha permanecido oculto bajo una concepción fundamentalmente estatocéntrica de los estudios en relaciones internacionales (Oddone y Luna Pont, 2019). La soberanía del Estado central constituía su esencia y principal razón de ser, y como tal necesitaba ser protegida de perforadores externos como internos.

Durante el siglo xx, y a medida que se consolidaba la disciplina de las relaciones internacionales, la premisa del Estado como actor unitario y central del escenario internacional -esgrimida principalmente por la escuela realista- comenzaba a enfrentar numerosos cuestionamientos. La creciente interdependencia económica, financiera y tecnológica, es un fenómeno que pone en evidencia esta pérdida de centralidad estatal, así como la necesidad de abordar las cuestiones internacionales desde perspectivas complementarias, tanto desde los actores como desde las dimensiones y temas. Estudiar los cambios en el sistema internacional constituye uno de los focos principales de la disciplina. Un autor sostiene que "los elementos constitutivos de las relaciones internacionales dan lugar a muchas especificidades y enfoques teóricos variados. Lo que provoca que la diversidad teórica de esta disciplina sea uno de sus puntos fuertes" (Halliday, 2002, p. 26).

A partir de los 60 las visiones estatocéntricas comenzaron a fragmentarse parcialmente; y dos décadas más tarde, las unidades subestatales fueron tímidamente incorporadas en el 
análisis de la política mundial. El grado de autonomía del actor subestatal frente al gobierno central será una de las características principales analizadas por el transnacionalismo a nivel global. García Segura (1993) hace hincapié en el papel del transnacionalismo como un parteaguas en la interpretación del actor internacional; una etapa que se corresponde con el fortalecimiento del pluralismo en la disciplina, también conocida como tercer debate o debate interparadigmático (Russell, 2010). Tal como se sostuvo: "No solo había más actores que el Estado, el Estado ya no era el Estado, sino que tenía que ser descompuesto en redes de burocracias, grupos de interés e individuos en una perspectiva pluralista" (Waever, 1996, p. 350).

$\mathrm{Al}$ complejizarse la interpretación de las relaciones internacionales y del papel que desempeñaban sus actores, las disciplinas que estudiaban los aspectos internos de los gobiernos, como el trabajo de las burocracias, fueron pronto incorporadas en el estudio de lo internacional. En este diálogo interdisciplinario, los aportes de Keohane y Nye a finales de los 70 fueron fundamentales ya que entendieron, al cuestionar al actor unitario de la realpolitik, el surgimiento de otros actores de naturaleza no estatal y la emergencia de nuevos temas en la agenda internacional. Keohane y Nye no realizaron una construcción teórica sobre el relacionamiento internacional de actores subnacionales, pero su entendimiento sobre el surgimiento de relaciones intergubernamentales y transnacionales, en paralelo a las relaciones interestatales, contribuyó con el desarrollo teórico de la proyección internacional subestatal. Con una nueva agenda internacional sin jerarquías, o con una jerarquía más difusa, tienen lugar múltiples canales de relacionamiento: interestatales, transgubernamentales o transnacionales. Los primeros son los canales estudiados por la escuela realista. Los canales transgubernamentales aparecen al flexibilizarse el supuesto realista que los Estados actúan coherentemente como unidades. Los canales transnacionales surgen cuando se disipa el supuesto realista que los Estados son las únicas unidades del sistema internacional. La evolución de esta interpretación fue generando numerosas líneas de investigación que impactaron sobre la reformulación del considerado hasta el momento paradigma hegemónico, como así también incluyeron los análisis críticos de la soberanía, como el de soberanías perforadas o soberanía compartida, hasta los estudios del isomorfismo institucional, entre otros.

James Rosenau (1990), desde una perspectiva social del liberalismo, subrayó la centralidad del transnacionalismo y del papel de personas y grupos sociales en la política internacional. El papel de estos actores no estatales es incentivado por el mayor acceso a la educación, las posibilidades que abren las nuevas tecnologías de la información y la pérdida del poder del Estado para controlar y regular todo lo que sucede al interior y exterior de las fronteras (Grasa, 2015). Rosenau contribuyó al estudio de la paradiplomacia como campo dentro de la teoría de las relaciones internacionales -aunque aún desde un lugar marginal-al plantear que entender la gobernanza global implica reconstruir procesos de gobernanza acumulados, que incluyen individuos, entes privados y públicos de diferentes niveles de gobierno (Rosenau, 1997). 
También desde la vertiente liberal, Moravcsik (1997) introdujo elementos teóricos que complejizaron la interpretación de la política internacional, subrayando la presencia de múltiples actores y preferencias al interior de los Estados. Desde la reformulación de la teoría liberal que propone, el Estado continúa ocupando un lugar central en la política internacional. No obstante, la construcción de las preferencias y las formas de representación como base de la política exterior permiten visibilizar actores al interior del Estado que el realismo suprime. Por esto, los aportes de Moravcsik (1997), aunque no hacen alusión a la paradiplomacia ni a los actores subnacionales, sí permiten analizar cómo la política exterior del Estado es el resultado de la puja entre intereses y preferencias de actores internos y habilita la desagregación de la política exterior, que no siempre es la expresión racional y unitaria de los intereses estatales.

El transnacionalismo permitió incluir una amplia gama de actores bajo el concepto de actor internacional (García Segura, 1992) y, a lo largo de los últimos años, se constituyó en uno de los encuadres privilegiados para el estudio de la paradiplomacia. Como concepto que emergió de la práctica internacional de gobiernos no centrales, el proceso de conceptualización de la paradiplomacia sigue estando abierto. Kuznetsov (2015) remarca la ausencia de marcos exploratorios para analizar la naturaleza multidimensional de la paradiplomacia que permitan una mayor comprensión. Como ha sido señalado "la mayoría de las obras se enfocan a describir el proceso, no a explicarlo, menos aún en buscar un fundamento conceptual" (Zeraoui, 2009, p. 64). Más allá de esta afirmación, y reconociendo que hay más casuística en el análisis que construcciones teórico-conceptuales, las diferentes interpretaciones sobre la paradiplomacia han sido enmarcadas en cinco grandes perspectivas (Oddone, 2016). Una primera, que explica la naturaleza y los cambios del actor internacional entendido en términos de unidades subestatales/subnacionales. En materia de atributos de las autoridades subnacionales que se internacionalizan se destacan: “a) el grado de autonomía, b) la capacidad o habilidad para movilizar recursos, ejercer influencias y alcanzar objetivos y c) la continuidad e importancia de las funciones que se desarrollan" (Russell, 2010, p. 84). Esta dimensión incluye, por lo general, el enfoque de las motivaciones para la acción internacional de los gobiernos subnacionales ${ }^{1} y$ los aspectos organizacionales y administrativos que se observan en las unidades geográficopolíticas del territorio (Calvento, 2015). Una segunda, que busca describir las complejidades en el diseño e implementación de una política exterior central (y en algunos casos nacional)

1 La clasificación más difundida fue presentada por Keating (1999), quien propuso tres categorías: económicas, culturales y políticas; luego el mismo autor en el 2003 incluyó las motivaciones secesionistas dentro de las políticas, como categoría separada y bajo el nombre de protodiplomacia. Kincaid (2003) incorporó una cuarta: los asuntos transfronterizos. Posteriormente, Michelmann (2009) utilizó dos de las tres motivaciones de Keating -la económica y la política- y agregó las altruistas. Álvarez (2016) produce una nueva clasificación, basada en dichos autores, agrupando las motivaciones en económicas, culturales, políticas, altruistas y fronterizas. 
que necesita reflejar los intereses subestatales/ subnacionales. Esta perspectiva se ha inspirado en los enfoques burocráticos y organizaciones que permitieron comprender mejor el proceso de policy making de la política exterior. Una tercera, más vinculada al enfoque económico de la paradiplomacia, que incluye la inserción internacional de las unidades subnacionales dentro de la economía política internacional y su impacto sobre las dinámicas locales (incluyendo las dinámicas urbanas). Una cuarta, centrada en el estudio de los procesos de integración regional como oportunidad para canalizar la actividad paradiplomática, en donde esta es incluso vista como respuesta a los nuevos recorridos de diseño de la política multinivel. Y una quinta perspectiva, muy ligada con la anterior, en donde se estudian los aportes de la paradiplomacia a los sistemas sectoriales de gobernanza global.

La inclusión de la paradiplomacia dentro del campo de estudio de las relaciones internacionales, por lo general, se ha dado en el marco de las cinco perspectivas enunciadas. Dependiendo del o la investigador/a, el espacio desde el cual se analiza, y el momento histórico-político, cada una de las perspectivas cobra mayor peso. Incluso, diferentes analistas de relaciones internacionales han trabajado sus investigaciones moviéndose dentro de las diferentes perspectivas de manera no excluyente. Este eclecticismo responde a la naturaleza transdisciplinar de la paradiplomacia, que se ha consolidado como práctica que atraviesa numerosos campos de acción de unidades subnacionales, y que puede ser abordado desde múltiples perspectivas. Todas las perspectivas, abonan a la comprensión de la paradiplomacia como una política pública local o regional, activa o reactiva, basada en un modelo de gestión con contenidos, metas y un espacio institucional definido. Desde la mirada del mainstream, la heterogeneidad de los casos dificulta todavía la elaboración de una teoría global (Gely, 2016). Pero, al mismo tiempo, el estudio de la paradiplomacia ha tendido a complejizarse cada vez más.

Calvento (2019) ha identificado las principales propuestas para medir el desempeño internacional de los actores subnacionales en América Latina, distinguiendo las que se enfocan en aspectos descriptivos de las que incorporan herramientas explicativas. Entre ellas, se destacan: Fronzaglia (2005) para el caso brasileño, Zubelzú (2008) para el argentino y Schiavon (2010) para el mexicano. Recientemente, Calvento (2019) se ha dado a la tarea de la generación del índice de participación internacional subnacional con base en la variabilidad y diversificación. Díaz Bay (2019) ha propuesto la medición del desempeño paradiplomático a través del Índice Sintético de Comportamiento Estratégico Subnacional en Argentina. A estos ejercicios de carácter nacional, podrían sumarse también los de carácter sectorial, como el de Lara (2019) para las ciudades o el de Álvarez (2016) para la paradiplomacia transfronteriza.

Estos avances han ayudado a percibir que ya no se trata de comprender el fenómeno paradiplomático como expresión de una acción (establecimiento de contactos, relaciones entre burocracias, reacción a la influencia de factores externos o frente a la complejidad del escenario internacional, entre otras), sino de estudiarlo como un tipo de política pública definida a partir de "entradas directas e indirectas de los 
gobiernos no centrales al campo de las relaciones internacionales y de su vinculación con la política exterior" (Duchacek, 1990, p. 15). Diferentes estrategias que podrían enmarcarse bajo el concepto de paradiplomacia han sido llevadas a cabo con el objetivo de aumentar los márgenes de autonomía de gobiernos subnacionales; generando una serie de modificaciones en las concepciones diplomáticas más tradicionales, legitimando su propio accionar y los intereses manifiestos en dinámicas multiactor y multinivel (Oddone y Luna Pont, 2019). Sin embargo, es importante remarcar que la inexistencia de una única vía para el desarrollo de la paradiplomacia implica que su diseño dependerá de la capacidad de concertación de las autoridades locales, la movilización de otros actores socios, los recursos disponibles, sus trayectorias de gobierno y la acumulación de otras experiencias, así como la posibilidad de imitar estructuras parecidas.

Los avances en los estudios de la paradiplomacia, en especial en América Latina, se han visto influidos por los aportes de investigadores de España, Canadá y otros países de la subregión, en principio, sobre todo de aquellos países de carácter federal, como Argentina, Brasil y México. El diálogo e intercambio entre investigadores de carácter informal, así como los congresos y seminarios académicos o de difusión e incidencia, los cursos de formación presenciales y en línea, el apoyo a proyectos de cooperación descentralizada o transfronteriza, el diálogo directo y la colaboración con redes de ciudades o regiones y las discusiones en redes sociales, han permitido la creación de una comunidad epistémica a escala transnacional. Las comunidades epistémicas cumplen un pa- pel fundamental en la agregación de intereses de investigación, socialización internacional de enfoques disciplinarios e intereses académicopolíticos. Gracias al apoyo de las tecnologías de la información y la comunicación, las comunidades epistémicas promueven propuestas de innovación social en políticas que, de lo contrario, permanecerían encerradas en grupos de investigación, organizaciones internacionales o gobiernos individuales sin producir efectos estructurales (Adler y Haas, 2009). En sentido contrario, si la comunidad epistémica tiene la capacidad de influir sobre procesos de toma de decisión en diferentes gobiernos en todos sus niveles, logrará una mayor materialización de sus propuestas transnacionales impactando en el diseño de políticas públicas.

\section{LA PARADIPLOMACIA COMO CAMPO DE ESTUDIO DE LAS RELACIONES INTERNACIONALES EN ARGENTINA: ¿INCIPIENTE CURRICULARIZACIÓN?}

Entre los centros de estudios latinoamericanos dedicados a investigar la inserción internacional de actores subnacionales, se pueden mencionar la División de Estudios Internacionales del Centro de Investigación y Docencia Económicas (Cide) y el Departamento de Estudios Internacionales del Centro Universitario de Ciencias Sociales y Humanidades (CUCsH) de la Universidad de Guadalajara, en México; el Centro de Estúdos de Cultura Contemporânea, el Centro Brasileiro de Relações Internacionais y el Laboratório de Análise Política Mundial de la Universidade Federal da Bahia, en Brasil; y el Centro de Estudios Políticos Internacionales del Grupo de Estudios Re- 
gionales de las Facultades de Ciencia Política y Gobierno y de Relaciones Internacionales en la Universidad del Rosario, en Colombia. De particular relevancia resulta el CUCSH por ser el responsable de la primera maestría en relaciones internacionales de los gobiernos y actores locales (MRIGYAL) en América Latina. La MRIGYAL inició su dictado en 2017 contando entre sus profesores a graduados de la Universidad de Guadalajara, Cide y la Universidad del País Vasco/Euskal Herriko Unibertsitatea y es un programa pionero en la región centrado en la profesionalización de recursos humanos en internacionalización de gobiernos locales.

Estos centros de investigación y docencia concentran sus análisis en la participación global de los gobiernos de las grandes ciudades y los avances realizados son, predominantemente, descriptivos, con algunas excepciones en los estudios del Cide y Cucsh. Aquellos que han buscado ahondar en las causas, se han concentrado en análisis monocausales, centrados en factores relativos al tamaño y ubicación de la ciudad, o en el marco jurídico que habilita o limita dicha performance, así como en el contexto interno y externo que favoreció tal desarrollo $^{2}$, elementos que no siempre explican las variaciones observadas al interior del universo de los gobiernos subnacionales.

En el caso argentino, uno de los centros pioneros en paradiplomacia es el Centro de Estudios en Relaciones Internacionales de Rosario (Cerir) de la Universidad Nacional de Rosario, Provincia de Santa Fe. Fundado en
1987, y dirigido inicialmente por Bruno Bologna, se integró por un conjunto de docentes de la Facultad de Ciencia Política y Relaciones Internacionales, estableciendo entre sus objetivos "diseñar, programar y poner en marcha estructuras al servicio del desarrollo económico, social y cultural de la región litoral y pampeana y su proyección al ámbito internacional", contando con proyectos de investigación acreditados que vienen generando conocimiento sobre la participación internacional de provincias argentinas y otros actores subnacionales.

La temática fue incorporada en el proyecto denominado "La Inserción de la República Argentina en el Sistema Internacional" aprobado por Conicet como Proyecto de Investigación en 2001, dirigido por Bruno Bologna. También en los siguientes proyectos grupales presentados al Programa de fomento a la investigación científica de la Universidad Nacional de Rosario: "La Inserción de la Argentina en el Sistema Internacional" (1996), dirigido por Bruno Bologna y codirigido por Miryam Colacrai; "Inserción Política y Económica de la Provincia de Santa Fe en el Mercosur" (1997), dirigido por Gladys Lechini y codirigido por Anabella Busso.

En el mismo, Graciela Zubelzú y Miryam Colacrai, realizaron relevantes aportes adaptando la teoría de las relaciones internacionales abocada al accionar contemporáneo de los actores subnacionales con base en la pionera conceptualización de la gestión internacional (Colacrai y Zubelzú, 1994; 1998; 2004) para el

\footnotetext{
2 Una excepción a dicha afirmación es el estudio elaborado por Graciela Zubelzú "El diseño institucional y los perfiles de gestión externa de las provincias argentinas" (2008).
} 
caso de provincias argentinas, la cual marcó un punto de diferenciación con las concepciones paradiplomáticas prevalecientes. Dicho enfoque fue presentado hace más de dos décadas en su primer artículo de aproximación a la temática (Colacrai y Zubelzú, 1994) publicado en Cuadernos de Politica Exterior Argentina, editados por Cerir desde 1985. Asimismo, Cerir, a través de su maestría en integración y cooperación internacional que se dicta en la Universidad Nacional de Rosario desde 1995, mantiene un constante tratamiento del tema por medio de su dictado; así como por la generación continua de tesis de posgrado dedicadas a la participación internacional subnacional.

Otra institución relevante es el Consejo Argentino para las Relaciones Internacionales (Cari), ubicado en la ciudad de Buenos Aires, y creado en 1978 como una institución académica privada sin fines de lucro. Entre sus objetivos establece el estatuto: "la promoción del estudio y la profundización científica de las relaciones internacionales, especialmente de la República Argentina, y los asuntos y problemas vinculados a ellas".

En 2001, justificado por el incremento de la participación de actores subnacionales, Cari crea en su interior el Comité de Provincias en el Plano Internacional dirigido oportunamente por el embajador Eduardo Iglesias e integrado por miembros del poder ejecutivo nacional, académicos y del sector privado, que busca ser un espacio de discusión y elaboración de propuestas sobre el accionar externo de las provincias y regiones argentinas y un espacio mul- tidisciplinario para estudiar distintos aspectos referidos a la economía y finanzas, comercio, infraestructura, medio ambiente, turismo y migraciones, entre otros temas (Rodríguez Giavarini, 2008).

Este Comité ha llevado adelante un conjunto de actividades que incluyen: reuniones con expertos, talleres de trabajo con funcionarios provinciales y nacionales, elaboración de informes y de documentos de trabajo, entre otras. Asimismo, se establecieron acuerdos con otras instituciones y organismos donde se destaca el proyecto de cooperación "Provincia y Relaciones Internacionales", entre Cari y el Programa de Naciones Unidas para el Desarrollo, que permitió relevar información sobre la gestión internacional de las provincias a través de diferentes informes y el libro Las provincias argentinas en el escenario internacional. Desafios y obstáculos de un sistema federal, compilado en el 2008 por Eduardo Iglesias, Valeria Iglesias y Graciela Zubelzú.

Producto de este trabajo y de su continuidad, Cari ha realizado una sistematización de los convenios internacionales celebrados por provincias argentinas. Para ello ha establecido acuerdos con diferentes provincias que permitieron un relevamiento, profundización y divulgación de la actividad internacional de estos actores subnacionales que en muchos casos se convierte en el único registro oficial de la actividad. Actualmente, se cuenta, aunque con limitaciones ${ }^{3}$, registros para la totalidad de las provincias argentinas.

3 Cari aclara que "en algunos casos el inventario de los acuerdos puede llegar a ser incompleto, en circunstancias especiales por imposibilidad de su ubicación o transcripción textual, en otros por alguna dificultad en provincias que 
En otro plano, se destaca el Centro de Estudios Interdisciplinarios en Problemáticas Internacionales y Locales (Ceipil), dirigido por José María Araya, que desempeña sus actividades en Tandil, Provincia de Buenos Aires, y desde hace una década se ocupa de la generación de conocimiento sobre el accionar de los municipios argentinos. Creado en 1998 en la Universidad Nacional del Centro de la Provincia de Buenos Aires (Unicen), dependiente de las Facultades de Ciencias Humanas y Ciencias Económicas, fue incorporado como Centro Vinculado y luego como asociado a la Comisión de Investigaciones Científicas del Gobierno de la Provincia de Buenos Aires. Tal como su denominación lo indica, desde Ceipil se desarrolla un especial compromiso con el estudio de las problemáticas internacionales contemporáneas y del entorno local/regional donde se inserta la universidad, teniendo en cuenta las interrelaciones y mutuos condicionantes existentes.

En ese sentido, realiza relevantes actividades de investigación a través de diferentes líneas de trabajo, una de las cuales se vincula a la participación internacional de gobiernos locales, que se ocupa de estudiar las diferentes estrategias, modalidades, causas y alcances de la participación y vinculación internacional que desempeñan distintos actores, en especial los municipios. Se trabaja en la temática desde 2010 a través de proyectos de investigación, transferencia, extensión y docencia.
En el marco de Ceipil se desarrollaron los siguientes proyectos presentados al Programa de Incentivos a Docentes Investigadores (Ministerio de Educación): "Actores y dinámicas en las estrategias de desarrollo local: el rol de los municipios como espacios de articulación para el desarrollo de estrategias de proyección nacional e internacional”, dirigido por José María Araya y codirigido por Mariana Calvento; "Espacios subnacionales y actores locales: dinámicas y procesos en el desarrollo de estrategias de participación internacional", dirigido por José María Araya y codirigido por Mariana Calvento; "Relaciones internacionales y actores subnacionales: dinámicas, estrategias y políticas en el interior de la Provincia de Buenos Aires”, dirigido por Mariana Calvento. También el proyecto "Actores y dinámicas en las estrategias de desarrollo local: el rol de los gobiernos municipales en la generación de políticas de gestión internacional”, financiado por la Agencia Nacional de Promoción Científica y Tecnológica y dirigido por Mariana Calvento.

Algunas de las principales contribuciones al tema se basan en el desarrollo de nuevos conceptos, entre los que se destacan el de política internacional subnacional (Maira, 2010) y la construcción del índice de participación internacional (Calvento, 2015; 2016), producto de los estudios realizados fundamentalmente sobre municipios intermedios de la Provincia de Buenos Aires; mientras otros de los aportes consignados revelan aproximaciones sobre

no incorporaron toda la documentación y finalmente, en el caso de las provincias de Neuquén y la Pampa que no se obtuvo un total acompañamiento de las autoridades de los ejecutivos provinciales consultados o requerida su colaboración”.

OASIS, ISSN: 1657-7558, E-ISSN: 2346-2132, N³2, Julio - Diciembre de 2020, pp. 63-84 
la articulación que establecen los gobiernos locales con actores territoriales en el diseño e implementación de estrategias de inserción internacional (Nicolao y Piersanti, 2019).

Es importante mencionar la Unidad de Investigación sobre Gobiernos Locales del Departamento de Economía y Administración de la Universidad Nacional de Quilmes (UNQ), dirigida por Daniel Cravacuore, que establece como objetivo el desarrollo "de investigación, docencia, extensión y transferencia sobre la problemática de la política y gestión de los gobiernos locales". En ese sentido, profesionales de UNQ han colaborado en actividades de investigación ${ }^{4}$ y asesoramiento sobre la actividad internacional de los actores subnacionales a organismos nacionales, regionales e internacionales. En el marco de actividades propuestas por UNQ, se conformó la Red InterMuni que reúne a los responsables de cooperación internacional de municipios argentinos. Desde este espacio dirigido por Cravacuore y Oddone se realizó un primer diagnóstico sobre la institucionalización de las áreas de relaciones internacionales en los municipios nacionales, destacándose que:

- Existe una gran variedad de niveles y formas de institucionalización del área de relaciones internacionales en los municipios. En algunos casos, dependen de los departamentos de desarrollo económico, hacienda, modernización del Estado o de gobierno; en otros casos no existe una estructura institucional que contenga dichas actividades o bien está en etapa de formación. El personal abocado a estas tareas es escaso y muchas veces no está dedicado de tiempo completo a estas actividades. La mayoría de las áreas no cuentan con un presupuesto asignado.

- Las actividades de cooperación internacional son interdisciplinarias. Ello requiere el trabajo coordinado entre diferentes áreas del municipio y la colaboración de técnicos, dependiendo de la naturaleza del programa, proyecto o actividad.

- La falta de recursos humanos y financieros es la principal dificultad para desarrollar acciones de cooperación internacional municipal. Existen áreas de oportunidad a mejorar en cuanto a la profesionalización del personal, sobre todo en cuestiones vinculadas a la formulación y seguimiento de proyectos bajo una lógica intermunicipal. Recientemente, Cravacuore (2019) ha publicado una recopilación sobre la intermunicipalidad en Argentina y sus vínculos con la cooperación internacional.

Otra experiencia de creciente importancia es la Unidad Ejecutora en Ciencias Sociales Regionales y Humanidades (Cisor) que funciona en la Universidad Nacional de Jujuy (Unju), se desarrollan líneas de investigación que se concentran en el estudio de las relaciones entre unidades subnacionales de frontera desde una perspectiva histórica y de relaciones interna-

\footnotetext{
4 Proyecto financiado por UNQ: "Políticas municipales de internacionalización económica como instrumento de
} desarrollo productivo local en Argentina” (2004-2007). 
cionales contemporáneas. En la primera línea se pueden mencionar los proyectos de "Dinámicas sociales, económicas y culturales en la historia de la frontera argentino-boliviana: La Quiaca-Villazón (1900-1930)”, dirigido por Ana Teruel. Una segunda línea en proceso de consolidación aborda la gestión internacional de las provincias en un escenario internacional en reconfiguración, en particular con la emergencia de China como socio de relevancia en el desarrollo del sector energético en Argentina, Bolivia y Chile (Juste, 2017).

Estas instituciones, mencionadas en un listado no excluyente, suelen apoyarse también en otros centros de investigación, espacios de análisis, grupos de interés o think tank que potencian con sus estudios el desarrollo de la paradiplomacia entre los que se destacan: Paradiplomacia.org, el Centro de Implementación de Políticas Públicas para la Equidad y el Crecimiento, y la Fundación Centro de Estudios Internacionales Contemporáneos, entre otros.

Paralelo al desarrollo de estos centros de investigación se registra, como se ampliará más adelante, un importante conjunto de estudios que amplían la visión sobre la política internacional subnacional donde adquieren una relativa primacía las investigaciones realizadas a nivel provincial (Dalla Via, 2004; De Marsilio, 2006; Castro y Saslavsky, 2009) por sobre las que ponen su eje en gobiernos locales o municipales (González, 2003; Dabat, 2004; Cippec, 2012), a excepción de la Red Mercociudades, Red de Ciudades del Mercosur, que ha sido ampliamente estudiada (Petrantonio, 2003; Oddone, 2008; Granato y Oddone, 2008; Granato y Oddone, 2010; 2019, Alvaredo, 2019). Estos trabajos constatan la existencia de estrategias de gestión internacional en la generalidad de los gobiernos provinciales de Argentina, en los gobiernos de las grandes ciudades como Buenos Aires y Rosario, así como en capitales provinciales como Córdoba y el papel de las ciudades en la integración regional. Como se dijo, la casuística basada en experiencias individuales, municipales o provinciales, prima sobre otros análisis ya que, en menor medida, se han detectado trabajos que analizan ambos actores (provincias y ciudades) en conjunto o de manera comparada (De Marsilio, 2006; Vicchi, 2006).

En cuanto a los proyectos que actualmente se ejecutan con apoyo del Consejo Nacional de Investigaciones Científicas y Técnicas (Conicet), que presentan un vínculo directo o tangencial con la paradiplomacia, se concentran en enfoques desde las relaciones internacionales, historia y geografía, y antropología. En el primer grupo encontramos los siguientes: 1. Posdoctoral: "Grandes potencias y gestión transnacional de las provincias argentinas. Oportunidades y desafíos de la complementariedad con China en el desarrollo energético local y sus consecuencias sobre las comunidades locales (2014-2018)", en Unju-Cisor; 2. Doctoral: "El uso de la paradiplomacia en las unidades subnacionales argentinas para el acceso a créditos internacionales entre 1991 y 2011", en la Universidad Nacional de La Rioja; 3. Doctoral: "Diseño e implementación de políticas ambientales en los municipios del área metropolitana de la provincia de Mendoza (1992-2015)", en la Universidad Nacional de San Martín.

Desde la segunda línea, historia y geografía, se encuentran dos proyectos que tan- 
gencialmente trabajan la paradiplomacia. El primero sobre "Regiones y fronteras en perspectiva multiescalar. Agentes sociales y prácticas espaciales en la construcción de un mundo segregado, excluyente y fragmentado", en la Universidad de Buenos Aires. El segundo sobre "Desarrollo turístico y cooperación transfronteriza. Una comparación entre la triple frontera de Iguazú (Argentina-Brasil-Paraguay) y de la Circumpuna (Argentina-Bolivia-Chile)", en el Instituto Multidisciplinario de Historia y Ciencias Humanas. Por último, desde los estudios de seguridad, se inscribe el proyecto "Etnografía de los mercados ilegales y de la seguridad pública en la frontera argentina con Brasil y Paraguay", con doble dependencia de Conicet y la Universidad Nacional de Misiones.

Pasando de la investigación a la docencia, se constata que el tratamiento de los contenidos programáticos vinculados a la paradiplomacia en carreras vinculadas a las relaciones internacionales encuentra diferentes formas de análisis y enseñanza. Para esta investigación de carácter exploratorio se ha accedido a programas de grado y posgrado en relaciones internacionales de las siguientes universidades en Argentina: Universidad Nacional de Rosario, Universidad Nacional de San Martín, Universidad Nacional del Centro de la Provincia de Buenos Aires, Universidad Nacional de Lanús, Universidad Nacional de Tres de Febrero, Universidad de Buenos Aires, Universidad Nacional de Córdoba, Universidad Nacional de La Plata, Universidad Nacional de Quilmes, Universidad Nacional de Villa María, Universidad del Salvador, Universidad Católica de Córdoba, Universidad Siglo 21, Universidad
Argentina de la Empresa, Universidad de San Andrés, Universidad de Belgrano, Universidad Abierta Interamericana, Universidad Católica de Santa Fe, Universidad del Centro de Estudios Macroeconómicos de Argentina, Universidad Austral, Universidad Torcuato Di Tella, Universidad de Palermo, Universidad Maimónides, Universidad del Congreso, Universidad Católica de Salta, Facultad Latinoamericana de Ciencias Sociales sede Argentina, Universidad de Bolonia Argentina.

En primer lugar, se observa que en las carreras de grado en relaciones internacionales la temática, por lo general, tiende a ser incluida en programas de alguna materia o asignatura obligatoria, principalmente aquellas ligadas al análisis de la política exterior, y también en seminarios optativos, pero no ha sido incluida como una materia con entidad propia. Esto puede constatarse en la mayoría de las licenciaturas dictadas en universidades públicas, como la Universidad Nacional de Rosario, de San Martín y del Centro de la Provincia de Buenos Aires, y en algunas universidades privadas, como la Universidad del Salvador, Católica de Córdoba y Siglo 21 (Cuadro N ${ }^{\circ}$ 1). Esta cuestión puede estar asociada a la alta concentración de la enseñanza de las relaciones internacionales en universidades privadas en la Ciudad Autónoma de Buenos Aires, lo que ha llevado a una relativa invisibilidad de la temática, con la excepción de la Universidad de Belgrano, mientras que las carreras que son dictadas en el interior del país la reflejan a través de diferentes modalidades, destacándose sobre todo el tema de la paradiplomacia transfronteriza en las universidades de frontera de Argentina y los países del Cono Sur, 


\section{Cuadro $n^{\circ} 1$ \\ Carreras de grado de relaciones internacionales que incorporan contenidos programáticos sobre paradiplomacia}

\begin{tabular}{|c|c|c|}
\hline \multicolumn{3}{|c|}{ Universidades públicas } \\
\hline Carrera & Universidad & Ubicación dentro de la malla curricular \\
\hline $\begin{array}{l}\text { Licenciatura en Relaciones } \\
\text { Internacionales }\end{array}$ & $\begin{array}{l}\text { Universidad Nacional de } \\
\text { San Martín }\end{array}$ & Asignatura obligatoria Análisis de politica exterior*. \\
\hline $\begin{array}{l}\text { Licenciatura en Relaciones } \\
\text { Internacionales }\end{array}$ & $\begin{array}{l}\text { Universidad Nacional del } \\
\text { Centro de la Provincia de } \\
\text { Buenos Aires }\end{array}$ & Asignatura obligatoria Politica exterior argentina*. \\
\hline $\begin{array}{l}\text { Licenciatura en Relaciones } \\
\text { Internacionales }\end{array}$ & $\begin{array}{l}\text { Universidad Nacional de } \\
\text { Rosario }\end{array}$ & $\begin{array}{l}\text { Asignatura obligatoria Politica internacional } \\
\text { argentina* y asignatura optativa Seminario sobre } \\
\text { internacionalización de ciudades y regiones* (también } \\
\text { para la carrera de la Licenciatura en Ciencias Políticas). }\end{array}$ \\
\hline \multicolumn{3}{|c|}{ Universidades privadas } \\
\hline $\begin{array}{l}\text { Licenciatura en Relaciones } \\
\text { Internacionales }\end{array}$ & Universidad del Salvador & Asignatura obligatoria Teoría y práctica diplomática. \\
\hline $\begin{array}{l}\text { Licenciatura en Relaciones } \\
\text { Internacionales }\end{array}$ & $\begin{array}{l}\text { Universidad Católica de } \\
\text { Córdoba }\end{array}$ & $\begin{array}{l}\text { Asignaturas obligatorias Análisis de politica exterior* y } \\
\text { Derecho diplomático y consular*. }\end{array}$ \\
\hline $\begin{array}{l}\text { Licenciatura en Relaciones } \\
\text { Internacionales }\end{array}$ & Universidad Siglo 21 & Asignatura obligatoria Economía politica internacional. \\
\hline $\begin{array}{l}\text { Licenciatura en Relaciones } \\
\text { Internacionales }\end{array}$ & Universidad de Belgrano & $\begin{array}{l}\text { Asignaturas obligatorias Introducción a las relaciones } \\
\text { internacionales*, Teoría de las relaciones internacionales* } \\
\text { y Teoría y práctica diplomática y consular*. }\end{array}$ \\
\hline $\begin{array}{l}\text { Licenciatura en Relaciones } \\
\text { Internacionales }\end{array}$ & $\begin{array}{l}\text { Universidad del Centro de } \\
\text { Estudios Macroeconómi- } \\
\text { cos de Argentina }\end{array}$ & Asignatura obligatoria Relaciones internacionales*. \\
\hline $\begin{array}{l}\text { Licenciatura en Estudios } \\
\text { Internacionales }\end{array}$ & $\begin{array}{l}\text { Universidad Torcuato Di } \\
\text { Tella }\end{array}$ & $\begin{array}{l}\text { Asignatura optativa Procesos politicos y gobierno } \\
\text { subnacional. }\end{array}$ \\
\hline $\begin{array}{l}\text { Licenciatura en Relaciones } \\
\text { Internacionales }\end{array}$ & $\begin{array}{l}\text { Universidad Católica de } \\
\text { Salta }\end{array}$ & Asignatura obligatoria Derecho constitucional. \\
\hline
\end{tabular}

* Programa disponible en línea. Nota: Se registran solo las carreras que explícitamente incluyen el término paradiplomacia o alguno de sus sinónimos. Fuente: Elaboración propia con base en planes de estudios de las universidades relevadas. 
en particular, en Brasil y Chile. Un caso para destacar entre las universidades públicas es la Licenciatura en Desarrollo Local - Regional de la Universidad Nacional de Villa María, en la Provincia de Córdoba. Esta carrera, que se aboca a las problemáticas propias de los actores subnacional en sus diversas dimensiones incluye la gestión internacional subnacional entre los contenidos del plan de estudios.

La paradiplomacia aparece en las carreras de grado vinculada a los contenidos troncales de las licenciaturas en materias como relaciones internacionales, teoría de relacionales o en aquellas que analizan los problemas y desafíos del mundo contemporáneo, la política exterior argentina o el rol de la Argentina en la agenda global. En un único caso la paradiplomacia aparece vinculada al estudio del derecho constitucional y el federalismo argentino. Es de destacar la ausencia de este contenido en los talleres de prácticas profesionales. Esta cuestión resulta llamativa ya que crecientemente quienes se gradúan en relaciones internacionales buscan su inserción laboral en oficinas o departamentos de cooperación internacional o relaciones internacionales de carácter municipal o provincial.

En segundo lugar, al analizar las carreras de posgrado, la temática adquiere otra relevancia ya que se contempla en asignaturas $\mathrm{y}$ seminarios obligatorios $\mathrm{u}$ optativos como materia independiente en un importante número de maestrías y doctorados, vinculados a las relaciones internacionales (Cuadro $\mathrm{N}^{\circ} 2$ ).

La temática es incorporada en asignaturas obligatorias vinculadas a los nuevos procesos del escenario internacional, a las teorías de relaciones internacionales y de la integración regional, a la política internacional y nacional, siendo abordada -principalmente- a través de conceptos relativos a la paradiplomacia, internacionalización territorial, gestión externa o cooperación internacional, con particular énfasis en la cooperación internacional descentralizada.

Por otro lado, resulta relevante mencionar la existencia de posgrados que exceden el campo de las relaciones internacionales, pero que han agregado la temática como la Maestría en Gobierno Local de la Universidad Nacional de Quilmes, en el núcleo electivo Asociativismo Intermunicipal, la Maestría en Desarrollo Local de la Universidad Nacional de San Martín o la Maestría en Desarrollo Territorial de la Universidad Tecnológica Nacional en la Facultad Regional de Rafaela, en la Provincia de Santa Fe, que dan cuenta de la importancia de esta temática para el desarrollo local, la gestión pública y de los gobiernos locales. Asimismo, ha podido detectarse que la paradiplomacia es uno de los contenidos impartidos en el Programa de Estado y Políticas Públicas de Flacso Argentina en el Seminario de Posgrado Desarrollo, Políticas Públicas e Integración Regional que viene dictándose de manera sostenida desde 2013.

Por último, desde el desarrollo de los cuerpos teóricos de estas carreras de grado y posgrado se ha fortalecido el interés y desarrollo de trabajos finales de carrera y tesis de grado y posgrado que abarcan las diferentes temáticas vinculadas con la paradiplomacia, cuestión que buscará ser trabajada en una futura línea de investigación de los autores. 


\section{Cuadro $n^{\circ} 2$}

\section{Carreras de posgrado de relaciones internacionales que incorporan contenidos programáticos sobre paradiplomacia}

\begin{tabular}{|c|c|c|}
\hline \multicolumn{3}{|c|}{ Universidades públicas } \\
\hline Carrera & Universidad & Ubicación dentro de la malla curricular \\
\hline $\begin{array}{l}\text { Maestría en Integración y } \\
\text { Cooperación Internacional }\end{array}$ & $\begin{array}{l}\text { Universidad Nacional de } \\
\text { Rosario }\end{array}$ & $\begin{array}{l}\text { Seminario optativo II Cooperación } \\
\text { internacional, actores subnacionales y gobierno } \\
\text { locales*. }\end{array}$ \\
\hline $\begin{array}{l}\text { Maestría en Cooperación } \\
\text { Internacional }\end{array}$ & $\begin{array}{l}\text { Universidad Nacional de San } \\
\text { Martín }\end{array}$ & $\begin{array}{l}\text { Asignatura obligatoria Actores, políticas y } \\
\text { estrategias de la cooperación internacional para } \\
\text { el desarrollo } I^{*} \text {. }\end{array}$ \\
\hline $\begin{array}{l}\text { Maestría en Integración } \\
\text { Latinoamericana }\end{array}$ & $\begin{array}{l}\text { Universidad Nacional Tres } \\
\text { de Febrero }\end{array}$ & $\begin{array}{l}\text { Seminario III Actores sociales y unidades } \\
\text { subnacionales en procesos de integración } \\
\text { regional*. }\end{array}$ \\
\hline $\begin{array}{l}\text { Maestría en Procesos de } \\
\text { Integración Regional }\end{array}$ & Universidad de Buenos Aires & $\begin{array}{l}\text { Asignatura obligatoria Relaciones } \\
\text { internacionales e inserción internacional del } \\
\text { Mercosur. }\end{array}$ \\
\hline $\begin{array}{l}\text { Maestría en Relaciones } \\
\text { Internacionales }\end{array}$ & $\begin{array}{l}\text { Universidad Nacional de } \\
\text { Córdoba }\end{array}$ & $\begin{array}{l}\text { Seminario optativo Relaciones transnacionales } \\
\text { y paradiplomacia*. }\end{array}$ \\
\hline $\begin{array}{l}\text { Doctorado en Relaciones } \\
\text { Internacionales }\end{array}$ & $\begin{array}{l}\text { Universidad Nacional de } \\
\text { Rosario }\end{array}$ & $\begin{array}{l}\text { Asignatura obligatoria Teoría de las relaciones } \\
\text { internacionales*. }\end{array}$ \\
\hline $\begin{array}{l}\text { Doctorado en Relaciones } \\
\text { Internacionales }\end{array}$ & $\begin{array}{l}\text { Universidad Nacional de la } \\
\text { Plata }\end{array}$ & $\begin{array}{l}\text { Curso acreditable Paradiplomacia y politica } \\
\text { internacional de las regiones*. }\end{array}$ \\
\hline \multicolumn{3}{|c|}{ Universidades privadas } \\
\hline $\begin{array}{l}\text { Maestría en Relaciones } \\
\text { Internacionales }\end{array}$ & $\begin{array}{l}\text { Universidad Católica de } \\
\text { Santa Fe }\end{array}$ & $\begin{array}{l}\text { Seminario obligatorio Gestión externa de } \\
\text { actores subnacionales*. }\end{array}$ \\
\hline $\begin{array}{l}\text { Maestría en Estudios } \\
\text { Internacionales: }\end{array}$ & $\begin{array}{l}\text { Universidad Torcuato Di } \\
\text { Tella }\end{array}$ & $\begin{array}{l}\text { Asignaturas obligatorias Argentina } \\
\text { en el Sistema Mundial* y Cooperación } \\
\text { internacional*. }\end{array}$ \\
\hline $\begin{array}{l}\text { Doctorado en Relaciones } \\
\text { Internacionales }\end{array}$ & Universidad del Salvador & $\begin{array}{l}\text { Seminario obligatorio Problemas de politica } \\
\text { internacional*. }\end{array}$ \\
\hline
\end{tabular}

* Programa disponible en línea. Nota: Se registran solo las carreras que explícitamente incluyen el término paradiplomacia o alguno de sus sinónimos. Fuente: Elaboración propia con base en planes de estudios de las universidades relevadas. 


\section{REFLEXIONES FINALES}

"Lo que hoy denominamos la disciplina de las relaciones internacionales ha recorrido un largo trayecto histórico, antes de su consideración como disciplina científica en el marco de las ciencias sociales. Tan largo que empieza con las primeras consideraciones e interpretaciones de la realidad internacional. Interpretaciones teóricas de una práctica internacional que se remonta en el tiempo más allá de la aparición del Estado soberano y de la constitución del sistema europeo de Estados" (Del Arenal, 1981, p. 852). El estudio de los procesos internacionales, el estudio del cambio internacional, probablemente sea el foco principal de atención de las relaciones internacionales y como tal, los actores -ya sean protagonistas o marginales y en constante evolución- ocupan un interés y atención central para comprender cómo activan (o desactivan) procesos. Es decir, si tienen un papel más activo o reactivo ante el cambio como característica estructural de las relaciones internacionales. Comprender y estudiar la paradiplomacia es investigar sobre las motivaciones y acciones del actor subnacional. Como resultado "los cambios en las asunciones e interpretaciones epistemológicas que ayudan a formular y estructurar el entendimiento y la acción colectiva constituyen la noción más significativa de aprendizaje en relaciones internacionales" (Adler y Haas, 2009, p. 164).

La inexistencia de una única vía para el desarrollo de la paradiplomacia implica que su diseño dependerá de factores endógenos de las autoridades subnacionales (capacidad de concertación, movilización de otros actores socios, recursos humanos y financieros dispo- nibles, trayectorias de gobierno, conocimiento de la agenda global, capacidad de imitación, entre otros) como de factores exógenos basados en oportunidades internacionales a partir de modificaciones sustantivas en la estructura del sistema internacional. Hoy en día, con una estructura sistémica más sensible a los cambios y a la incertidumbre, la diversidad teórica de las relaciones internacionales es una de sus fortalezas como campo disciplinar, y los avances realizados hacia un multiparadigmatismo ponen de relieve que las relaciones internacionales constituyen una globalidad que jamás podrá ser analizada como tal (Oddone y Luna Pont, 2019).

La evolución de los estudios de caso de paradiplomacia hacia estudios comparados, y con mayor organicidad, ha logrado aprovechar las experiencias empíricas para iniciar procesos de capitalización, basados en las nuevas capacidades de agencia de los gobiernos subnacionales, la difusión de recorridos del tipo localcentral/global-local en el diseño de políticas y la coordinación de políticas transnacionales. Estos enfoques han sido caracterizados por Cornago (2019) como enfoques relacionales y han llamado la atención de investigadores que representan múltiples disciplinas (Kuznetsov, 2015) de las ciencias sociales. De igual forma, el estudio de la paradiplomacia ha pasado a formar parte sustantiva de las discusiones sobre las diplomacias públicas y nuevas diplomacias, permitiendo así canalizar "innovaciones intelectuales [que] ayudan a los gobiernos a redefinir sus expectativas (...) y a coordinar sus acciones" (Adler y Haas, 2009, p. 157) para la innovación política. 
Más allá de las controversias frecuentemente suscitadas, la paradiplomacia es hoy en día un campo vibrante de estudio en las relaciones internacionales (Cornago, 2019). Su creciente curricularización en la Argentina es fiel ejemplo de este mayor interés por una actividad internacional de los gobiernos municipales y provinciales que puede ser analizada desde diferentes perspectivas. No obstante, al analizar los programas de las asignaturas, se observa que -en la mayoría de los casos- la forma de inclusión de la paradiplomacia es relativamente desordenada e improvisada, acompañada de un escueto contenido bibliográfico sobre la temática y fuentes con escasas referencias a autores y obras claves. De allí que su curricularización presenta características ad hoc en cada casa estudio y en cada programa. El eclecticismo, osadamente se podría decir el multiparadigmatismo, parece estar servido entre los comensales y ser la característica del menú.

\section{REFERENCIAS}

Acharya, A. y Buzan, B. (eds.). (2010). Non-Western International Relations Theory. Perspectives on and beyond Asia. London: Routledge.

Acharya, A. y Buzan, B. (2019). The Making of Global International Relations: Origins and Evolution of IR at its Centenary. Cambridge: Cambridge University Press.

Adler, E. y Haas, P. (2009). Conclusión: las comunidades epistémicas, el orden mundial y la creación de un programa de investigación reflectivo. Relaciones Internacionales 12, 145-169.
Alvaredo, N. (2019). Mercociudades: una red estratégica para Asunción y otros municipios del Paraguay. Asunción: ISM y Municipalidad de Asunción.

Álvarez, M., Luna Pont, M. y Oddone, N. (eds.). (2019). América Latina global. Estudios regionales sobre paradiplomacia. Ciudad Autónoma de Buenos Aires: Untref.

Álvarez, M. (2016). Paradiplomacia en las relaciones chileno-argentinas: la integración desde Coquimbo y San Juan. Leiden: Universiteit Leiden.

Calvento, M. (2019). Política internacional subnacional y su medición: debates y propuesta para su abordaje en el caso de los municipios de Argentina. Perfiles Latinoamericanos 27(54), 1-31.

Calvento, M. (comp.). (2016). Gestión y politica internacional subnacional. El caso de los municipios del interior de la Provincia de Buenos Aires. Tandil: Ceipil-AnPCyT.

Calvento, M. (comp.). (2015). Procesos y actores en la gestión de la politica internacional subnacional. Tandil: Ceipil-Anрсут.

Calvento, M. (2015). Hacia un concepto multidimensional de la política y la gestión internacional subestatal. Procesos y actores en la gestión de la politica internacional subestatal. Tandil: CeipilANPCyT, 15-40.

Castro, L. y Saslavsky, D. (2009). Cazadores de mercados. Comercio y promoción de exportaciones en las provincias argentinas. Ciudad Autónoma de Buenos Aires: Cippec.

Centro de Implementación de Políticas Públicas para la Equidad y el Crecimiento. (2012). La cooperación internacional como herramienta para el desarrollo local. Ciudad Autónoma de Buenos Aires: Cippec.

Colacrai, M. y Zubelzú, G. (2004). Las vinculaciones externas y la capacidad de gestión internacional desplegadas por las provincias argentinas en la última 
década. Una lectura desde las relaciones internacionales. Ciudad Autónoma de Buenos Aires: Cari.

Colacrai, M. y Zubelzú, G. (1998). Capítulo xv: El creciente protagonismo externo de las provincias argentinas. La politica exterior argentina 19941997. Rosario: Cerir-UnR, 319-333.

Colacrai, M. y Zubelzú, G. (1994). Las provincias y sus relaciones externas ¿̨Federalización de la política exterior o protagonismo provincial en las relaciones internacionales? Cuadernos de Politica Exterior Argentina, 6. Rosario: Cerir-UnR.

Cornago, N. (2019). Paradiplomacy: From de Margins to the Mainstream. Presentado en Primer Encuentro de Estudios sobre Paradiplomacia e Internacionalización Territorial. Córdoba: Universidad Siglo 21. Cornago, N. (2010). On the Normalization of Sub-State Diplomacy. The Hague Journal of Diplomacy 5 , 11-36.

Cravacuore, D. (2019). Intermunicipalidad y cooperación internacional en la Argentina. América Latina global. Estudios regionales sobre paradiplomacia. Ciudad Autónoma de Buenos Aires: Untref, 175-198.

Dabat, G. (2004). Políticas locales de comercio exterior en la Argentina: Comercio y desarrollo desde una perspectiva endogenista. Documento de Investigación. Bernal: UNQ.

Dalla Vía, A. (2004). El marco jurídico e institucional para la gestión internacional de los actores subnacionales gubernamentales en Argentina. Integración y Comercio, 8(21), BID-Intal, 11-26.

De Marsilio, E. (2006). Aportes teórico-metodológicos para el estudio de las relaciones paradiplomáticas. Qué, quiénes, y cómo. Análisis del caso argentino. Serie Breviario en Relaciones Internacionales 5, CEA unc, 1-17.
Del Arenal, C. (1981). La génesis de las relaciones internacionales como disciplina científica. Estudios Internacionales, 2(4), 849-892.

Díaz Bay, J. (2019). La internacionalización, un comportamiento estratégico subnacional. Una propuesta de medición para el caso argentino. Aldea Mundo. Fronteras e Integración Regional,24(47), 71-87.

Duchacek, I. (1990). Perforated Sovereignties: Towards a Typology of New Actors in International Relations. Federalism in International Relations. The role of subnational units. Oxford: Clarendon Press, 1-33.

Fronzaglia, M. (2005). Unidades subnacionais: um estudo de caso sobre a cidade de São Paulo-de 2001 a 2004. Campinas: Unicamp.

García Segura, C. (1993). La evolución del concepto de actor internacional en la teoría de las relaciones internacionales. Papers: Revista de Sociología, 41, 13-31.

García Segura, C. (1992). La presència de les entitats politiques subestatals a les Relacions Internacionals. Barcelona: UAB.

Gely, M. (2016). Hacia una mejor conceptualización teórica de la proyección internacional de los gobiernos locales. Relaciones transfronterizas $y$ paradiplomacia en América Latina. Aspectos teóricos y estudios de casos. Santiago: RIL Editores y UNAP, 161-176.

González, J. (2003). Nuevas realidades de vinculación de las comunas en el ámbito internacional. Ciudad Autónoma de Buenos Aires: Fundación Exportar. Granato, L. y Oddone, N. (2008). Mercociudades: Red de Integración. Una nueva realidad en América Latina. Ciudad Autónoma de Buenos Aires: Capital Intelectual.

Grasa, R. (2015). Neoliberalismo e institucionalismo. La reconstrucción del liberalismo como teoría 
sistémica internacional. Teorías de las relaciones internacionales. Madrid: Tecnos, 97-125.

Halliday, F. (2002). Las relaciones internacionales en un mundo en transformación. Madrid: La Catarata.

Juste, S. (2017). Marco jurídico de la gestión internacional de las unidades subestatales. Un estudio comparado de Argentina, Bolivia y Chile. Derecho y Ciencias Sociales, 1(17), 226-246.

Keating, M. (1999). Regions and international affairs: Motives, opportunities and strategies. Regional \& Federal Studies, 9(1), 1-16.

Keating, M. (2003). Paradiplomacia y constitución de redes regionales. Revista Valenciana D'Estudis Autonòmics 36, 39-50.

Keohane, R. y Nye, J. (1977). Poder e interdependencia. La politica mundial en transición. Ciudad Autónoma de Buenos Aires: Gel.

Kincaid, J. (2003). Foreign Relations of Sub-national Units. Federalism in a changing world: Learning from each other. Montreal: McGill-Queen's University Press.

Knutsen, T. (2008). A lost generation? IR scholarship before World War I. International Politics, 45(6), 650-674.

Kuznetsov, A. (2015). Theory and Practice of Paradiplomacy. Subnational governments in international affairs. New York: Routledge.

Lara, R. (2019). La inserción de las ciudades en el medio internacional. Una revisión histórica, teórica y empirica desde las relaciones internacionales. Guadalajara: Universidad de Guadalajara.

Liu, T. y Song, Y. (2020). Chinese Paradiplomacy: A Theoretical Review. SAGE Open, 10(1), 1-14.

Luna Pont, M. (2019). De IULA a CGLU: Municipalismos internacionales, narrativas y momentos. América Latina global. Estudios regionales sobre paradiplomacia. Ciudad Autónoma de Buenos Aires: Untref, 51-92.
Michelmann, H. (2009). Introduction. Foreign relations in federal countries. Montreal: Forum of Federations, International Association of Centers for Federal Studies and McGill-Queen's University Press.

Moravcsik, A. (1997). Taking Preferences Seriously: A Liberal Theory of International Politics. International Organization 51(4), 513-553.

Nicolao, J. y Piersanti, A. (2019). Diversificación de actores locales en el escenario internacional: debates y reflexiones en torno a las organizaciones de migrantes. Presentado en I Encuentro de Reflexión sobre Relaciones Internacionales. Buenos Aires: Aeria - Umet.

Oddone, N. y Luna Pont, M. (2019). Avances disciplinarios en las relaciones internacionales: La definición de actor internacional en el estudio de la paradiplomacia. Relaciones Internacionales 92(2), 1-31.

Oddone, N. (2016). La paradiplomacia desde cinco perspectivas: reflexiones teóricas para la construcción de una comunidad epistémica en América Latina. Relaciones Internacionales 89(2), 47-81.

Oddone, N. y Granato, L. (2010). Mercociudades: la integración del tejido urbano en el Cono Sur. Las ciudades y los poderes locales en las relaciones internacionales contemporáneas. Granada: UIM, Aecid, Segib, Inap y Oidles, 337-362.

Oddone, N. (2008). La Red de Mercociudades: Globalización, integración regional y desarrollo local. Valencia: UPV.

Petrantonio, M. (2003). Innovando en la gestión local: la importancia de definir una política exterior local. En Anales del V Seminario Nacional de RedMuni: La reforma municipal pendiente. Perspectivas y prospectivas. Mendoza: Uncu.

Rodríguez Giavarini, A. (2008). Prólogo. Las provincias argentinas en el escenario internacional. Desafios $y$ 
obstáculos de un sistema federal. Ciudad Autónoma de Buenos Aires: Cari-pnud, 7-8.

Rosenau, J. (1997). Along the domestic-foreign frontier. Exploring governance in a turbulent world. New York: Cambridge University Press.

Russell, R. (2010). El Estado nación y los actores gubernamentales no centrales: una relación complementaria. La politica internacional subnacional en América Latina. Ciudad Autónoma de Buenos Aires: Libros del Zorzal, 83-106.

Schiavon, J. (2010). Las relaciones exteriores de los gobiernos estatales: El caso de México. La politica internacional subnacional en América Latina.
Ciudad Autónoma de Buenos Aires: Libros del Zorzal, 135-176.

Vicchi, A. (2006). La promoción de exportaciones en el sistema federal - Hacia un funcionamiento óptimo. Ciudad Autónoma de Buenos Aires: Uces.

Weaver, O. (1996). The rise and fall of the inter-paradigm debate. International Theory. Positivism and Beyond. Cambridge: Cambridge University Press, 149-185.

Zeraoui, Z. (coord.) (2009). Regionalismo y paradiplomacia. La politica internacional de las regiones. Puebla: ITESM y Montiel \& Soriano Editores. 